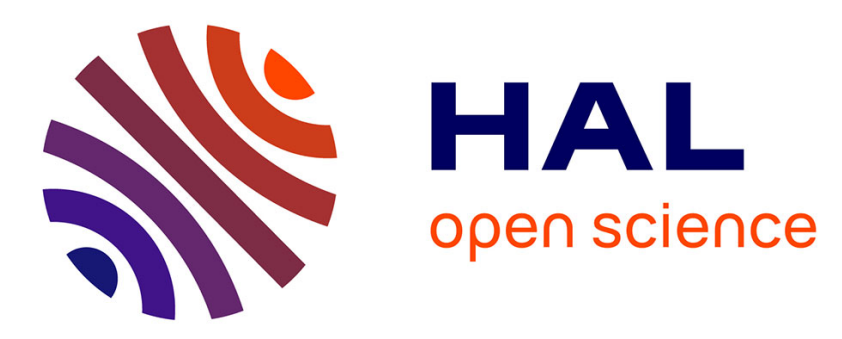

\title{
Large Eddy Simulation of Light-Round in an Annular Combustor With Liquid Spray Injection and Comparison With Experiments
}

\author{
Théa Lancien, Kevin Prieur, Daniel Durox, Sébastien Candel, Ronan \\ Vicquelin
}

\section{To cite this version:}

Théa Lancien, Kevin Prieur, Daniel Durox, Sébastien Candel, Ronan Vicquelin. Large Eddy Simulation of Light-Round in an Annular Combustor With Liquid Spray Injection and Comparison With Experiments. Journal of Engineering for Gas Turbines and Power, 2018, 140 (2), 10.1115/1.4037827 . hal-01744503

\section{HAL Id: hal-01744503 \\ https://hal.science/hal-01744503}

Submitted on 27 Mar 2018

HAL is a multi-disciplinary open access archive for the deposit and dissemination of scientific research documents, whether they are published or not. The documents may come from teaching and research institutions in France or abroad, or from public or private research centers.
L'archive ouverte pluridisciplinaire HAL, est destinée au dépôt et à la diffusion de documents scientifiques de niveau recherche, publiés ou non, émanant des établissements d'enseignement et de recherche français ou étrangers, des laboratoires publics ou privés. 


\title{
LARGE-EDDY SIMULATION OF LIGHT-ROUND IN AN ANNULAR COMBUSTOR WITH LIQUID SPRAY INJECTION AND COMPARISON WITH EXPERIMENTS
}

\author{
Théa Lancien ${ }^{\S}$, Kevin Prieur ${ }^{\ddagger}$, Daniel Durox ${ }^{\S}$, Sébastien Candel ${ }^{\S}$, Ronan Vicquelin ${ }^{\S}$ \\ $\S$ Laboratoire EM2C, \\ CNRS, CentraleSupélec, Université Paris-Saclay \\ 92295 Châtenay-Malabry cedex, France \\ \$Safran Tech, E\&P, \\ Chateaufort \\ 78772 Magny-Les-Hameaux, France
}

The light-round is defined as the process by which the flame initiated by an ignition spark propagates from burner to burner in an annular combustor, eventually leading to a stable combustion. Combining experiments and numerical simulation, it was recently demonstrated that under perfectly premixed conditions this process could be suitably described by large eddy simulation (LES) using massively parallel computations. The present investigation aims at developing light-round simulations in a configuration that is closer to that found in aero-engines by considering liquid nheptane injection. The large-eddy simulation of the ignition sequence of a laboratory scale annular combustion chamber comprising sixteen swirled spray injectors is carried out with a mono-disperse Eulerian approach for the description of the liquid phase. The objective is to assess this modeling approach of the two-phase reactive flow during the ignition process. The simulation results are compared in terms of flame structure and light-round duration to the corresponding experimental images of the flame front recorded by a high-speed intensified CCD camera and to the corresponding experimental delays. The dynamics of the flow is also analyzed to identify and characterize mechanisms controlling flame propagation during the light-round process.

Keywords: Two-phase flow, Large-Eddy Simulation, Lightround, Ignition, Eulerian-Eulerian approach, Annular combustor.

\section{NOMENCLATURE}

$B \quad$ Spalding transfer number

$D_{20} \quad$ Mean surface diameter

$D_{30}$ Mean volume diameter

$D_{32}$ Sauter Mean Diameter (SMD)
TFLES Thickened Flame model for LES

UQ Uncertainty Quantification

Polynomial Chaos expansion order

Polynomial Chaos

Gaseous laminar burning velocity

$k$-th species mass fraction

Progress variable

Droplet diameter

Mass flowrate

Axial gaseous velocity

Bulk velocity

Gas thermal diffusivity

Gas density

Liquid density

Light-round duration

Equivalence ratio

PCE

Uncertain parameters for the PCE

Large Eddy Simulation

Number Density Function

Polynomial Chaos Expansion
Efficiency function

Two-phase laminar burning velocity

$k$-th species mass fraction at equilibrium

Polynomial coefficient in the PCE

Injected droplet diameter in the simulations

Physical or numerical variable approximated through

\footnotetext{
*Address all correspondence to this author: thea.lancien@ centraliens.net
} 


\section{Introduction}

Ignition is one of the central issues in the development of modern combustion systems and engineering design has to ensure that it is reliable and safe. It is particularly critical in the case of gas turbines and aero-engines, where an additional complication arises from the presence of multiple injection devices. A successful ignition process follows three main stages [1]: (1) The igniter generates a spark that initiates the formation of a kernel of burnt gases; (2) If the kernel has enough energy to enable its spreading, the burnt gases volume increases until the closest fuel injector is ignited, creating a first stabilized flame; (3) The flame propagates from ignited burner to neighboring non-ignited burner until a flame is stabilized in the vicinity of all injectors and a steady-state is established. While there are many studies of the first two stages, the last step, also designated as lightround for a flame propagating in an annular chamber, is less well investigated in the literature mainly because of its geometric complexity. A better understanding of this process is however relevant when considering ignition delay or success and failure of the combustor's ignition completion. In this context, the pioneering simulation of the light-round in an annular helicopter chamber carried out in [2] demonstrates the new capabilities of LES but without detailed comparison with experiments. The ignition of a linear array combustor comprising five injectors has been studied experimentally and numerically in [3]. This however only partially reproduces processes taking place during light-round and for example does not account for remote interactions of the flame fronts nor does it provide information on the later part of the process where the two flames formed at the spark plug collide head on. It is important to operate in an annular enclosure to be representative of reality. This has led to the development of model scale facilities allowing such investigations. One of these installations designated as MICCA and designed at EM2C for combustion dynamics studies has provided full optical access allowing the systematic experiments reported in [4]. These have included high-speed visualizations of the flame propagation process during the ignition sequence, determination of the light-round delay and evolution of this delay with injection conditions. Level set G-equation simulations [4] of the light-round initial phase highlighted the key role of the flame turbulent burning velocity enhanced by the burnt gases volumetric expansion. The MICCA experiments carried out under premixed propane air injection were simulated with massively parallel large-eddy simulations $[5,6]$. Detailed comparisons with experiments indicated that the LES framework was able to retrieve many of the features observed in the experiment like the flame structures and configurations during the process, the light-round delay and evolution of the heat release rate in the system. Two combustion models were also compared with experimental data in [6]. Another set up with a more limited optical access and initially designed to study self-excited combustion instabilities $[7,8]$ was also used to analyze the lightround process $[9,10]$ under premixed and non-premixed conditions.

With the noticeable exception of [2], all the previous investigations were carried out under gaseous conditions, while aero-engine combustors are operated with liquid fuel injected as a spray. In order to be more representative of real ignition conditions, it is important to deal with this additional complexity and examine a situation where the fuel is injected as a spray of droplets by aerodynamic swirlers. It is then appropriate to use, once again, a combined experimental and numerical approach to obtain insights on this process. This has led to the design of the MICCA-Spray annular combustor which operates with swirl injectors fed with liquid fuel. Light-round experiments carried out in this new facility [11] allow comparisons with detailed simulations. The objective of the present article is to describe the methodology adopted to develop these simulations and to compare results of massively parallel LES calculations with the available data.

One important aspect is that of the numerical modeling of the liquid spray. This gives rise to a set of issues: (1) The description of the atomization process that generates small droplets would need computational resources that are not available in the context of the present study. A model is therefore needed for the description of the resulting dispersed liquid phase that is injected in the simulations, (2) The evolution of the droplet mist has to be described at a computational cost consistent with the available resources. Two main approaches exist for the large eddy simulation of two-phase reacting flows, both relying on mesoscopic models [12]: the Eulerian-Eulerian approach, where moments of the number density function (NDF) are transported [13,14] and the Eulerian-Lagrangian model where a set of individual particles are being tracked [15-17]. In the Eulerian-Lagrangian framework, it is relatively easy to account for polydispersion, while the Eulerian-Eulerian approach requires an additional set of transport equations for each moment and/or class of particle sizes, notably increasing the computational cost. It is however difficult to simulate a transient ignition process with the Lagrangian methods because they demand the use of a computational load balancing that is complex to handle on parallel machines [18]. This is expected to be difficult in a situation where the flame fronts propagate in the annular chamber, requiring a dynamical treatment of computational load balancing between ignited and un-ignited injectors. As a compromise between cost and accuracy, and considering the computational tools and resources available, the present study focuses on the simulation of MICCA-Spray light-round sequence in a mono-disperse Eulerian-Eulerian framework.

This study is organized as follows. A brief description of the MICCA-Spray experimental configuration is first provided. The corresponding numerical set-up and physical modeling retained for the large eddy simulations are then described. Comparisons with experimental data are then presented along with first analyses of the large data volume generated by these calculations. 


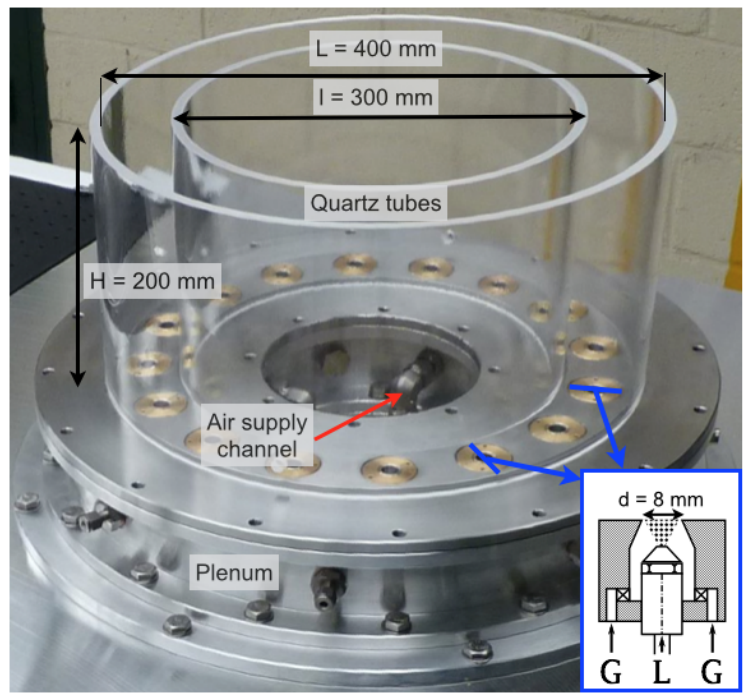

Fig. 1. Direct view of the MICCA-Spray combustion chamber. the sketch at the bottom right represents a cut of the swirler unit showing the gaseous ( $G$ arrows) and liquid ( $L$ arrow) injection.

\section{Experimental configuration}

The experimental set-up, sensors and imaging instrumentation and ignition procedure are successively described.

\subsection{Set-up}

The MICCA-Spray set-up (Fig. 1) is a laboratory-scale combustor representative of a helicopter combustion chamber [11]. It is composed of sixteen swirled injectors placed in the backplane of an annular chamber made of two cylindrical coaxial quartz walls. These injectors are fed with air and liquid fuel (n-heptane). The air is injected at ambient pressure and temperature into an annular plenum by eight identical channels and then into the chamber through the sixteen injectors. The fuel is fed to sixteen simplex atomizers placed after the swirlers (see the sketch in Fig. 1) and positioned with a $6 \mathrm{~mm}$ recess with respect to the chamber backplane. The measured swirl number equals 0.68. The inner and outer quartz walls that compose the chamber have a diameter of, respectively, $300 \mathrm{~mm}$ and $400 \mathrm{~mm}$ and are both $200 \mathrm{~mm}$ long. The burnt gases are then exhausted into the atmosphere. The MICCA-Spray chamber is able to reach a maximum power of $120 \mathrm{~kW}$.

The association of one swirl device and one sixteenth of the volume of the chamber forms a periodic pattern that is repeated sixteen times to make up the whole set-up. The chamber is divided into two halves, designated as $\mathrm{H}+$ and $\mathrm{H}-$, that are defined in Fig. 2, which also identifies the sixteen sectors. Sector S0 is the one where the ignition is initiated and sector S8 is located on the opposite side, where the flame front merging is expected. The sectors in between are respectively labelled $S_{i}$ if on the $\mathrm{H}+$ side of the chamber and $S_{-i}$ on the H- side of the chamber, with $i$ ranging from 1 to 7.

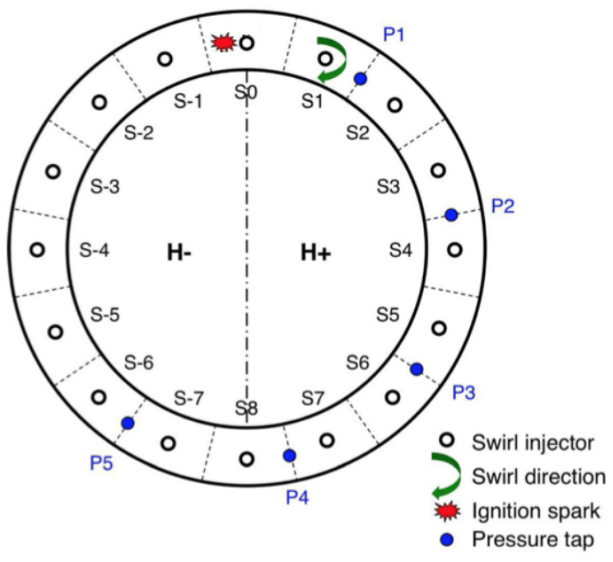

Fig. 2. Schematic top view of the MICCA-Spray backplane showing the swirlers positions, pressure taps and spark plug, extracted from [5].

\subsection{Sensors and imaging instrumentation}

The walls of the chamber are fully transparent, allowing an optical access to the flame. The evolution of the flame during the ignition sequence is recorded by a highspeed intensified complementary metal-oxide semiconductor $(\mathrm{CMOS})$ camera, with a resolution of $512 \times 512$ pixels $^{2}$. The frame rate and shutter speed are respectively $6000 \mathrm{~Hz}$ and $166 \mu \mathrm{s}$. The camera is sensitive to radiation in the visible range, down to a wavelength $\lambda=200 \mu \mathrm{m}$. An intensifier is used to enhance the signal, with a gain that remains constant during the light-round process. The camera is placed on the opposite side of the spark plug, at a distance of 3.1 $\mathrm{m}$ away from the chamber axis and $1.12 \mathrm{~m}$ above its backplane. MICCA-Spray also features five pressure taps, placed as shown in Fig 2. Each pressure tap is connected to a microphone flush mounted in a waveguide, with an acquisition frequency of $32,768 \mathrm{~Hz}$.

In addition to MICCA-Spray, experimental data on the gaseous flow and the liquid spray is derived from a singleburner set-up, composed of a unique swirler unit identical to those used in MICCA-Spray placed in a cylindrical chamber providing the same level of confinement as that prevailing in the annular chamber. Further details may be found in Ref. [11]. A Phase Doppler Anemometry system (PDA) is used to measure the gas and droplets velocity profiles as well as the droplet sizes. Measurements are carried out at different distances from the injector exhaust section so as to provide sufficient data for numerical validation.

\subsection{Experimental ignition procedure}

To compare experimental and numerical results, it is primordial to achieve similar conditions. In this respect, one central issue is the numerical treatment of the boundary conditions. The impact of temperature on the ignition delay is significant, as will be seen later on. As a first approximation, the choice is made to neglect heat transfer at the walls in the numerical simulation and assume that the walls are adiabatic. In order to match as closely as possible this condition 
experimentally, one must reduce the difference in temperature between the burnt gases and the walls. As in previous numerical studies [5], the ignition is then examined under relight conditions, when the walls are at a temperature corresponding to the steady-state stabilized flame regime. With such hot walls, heat losses are minimal. In practice, MICCASpray is first operated for approximately 10 minutes until the steady-state is reached. Liquid supply is then turned off and immediately on again. In practical conditions, ignition is initiated as soon as fuel begins to flow in the chamber. However, the transient ramping of the fuel mass flow rate to the nominal value is not well controlled and this would complicate comparisons of ignition simulations since the lightround would then occur in an unknown fuel-air mixture. To allow a consistent comparison, the ignition in experiments is initiated once a permanent regime has been reached for the unburnt two-phase mixture in the chamber. In practice, this requires a few seconds, a duration that exceeds the residence time in the combustor, so that the burnt gases are fully evacuated from the chamber and replaced by the fresh two-phase mixture.

\section{Numerical configuration and validation}

The numerical set-up, single-burner validation and droplet diameter selection are successively considered.

\subsection{Description of the numerical set-up}

It is natural to first review the main elements of the numerical framework. These include the reactive flow solver, physical models, computational domain and boundary conditions.

Simulations are carried out with the AVBP [19] code, jointly developed by Cerfacs and IFPEN, that solves the three-dimensional compressible Navier-Stokes equations for reactive flows on unstructured meshes. This software is based on a two-step Taylor-Galerkin weighted residual central distribution scheme, third order in time and space [20], for both gaseous and liquid phases. The subgrid scale turbulence is described with the Wall Adapting Local Eddy model [21]. As indicated previously, the liquid phase is modeled using an Eulerian-Eulerian mono-disperse approach. Six transport equations for the liquid mass concentration, the volumetric number of droplets, the three components of the liquid momentum and the liquid enthalpy [22] are added to the gaseous phase equations. The evaporation of the uniform temperature droplets is described by the AbramzonSirignano model [23].

The n-heptane chemistry is described using the two-step mechanism derived in [24, 25], obtained using the methodology proposed in [26] to reproduce the laminar flame speed and the adiabatic temperature for a wide range of operating conditions. The large eddy simulation combustion model describing the unresolved small scale flame structures and the propagation of the flame and its interaction with turbulence relies on the Thickened Flame model (TFLES [27,28]). This model is based on the artificial thickening of the flame fronts so as to be able to resolve it on the mesh, coupled with an efficiency function $\mathcal{E}$ that accounts for the subgrid scale flame wrinkling lost by making use of the thickening procedure. The thickening of the flame front is defined dynamically according to [29] so that the combustion model only impacts the flame region. This is used in combination with the efficiency function derived in [30].

As in previous simulations of premixed gaseous conditions, the numerical domain, shown in Fig. 3, is composed of the exact geometry of MICCA-Spray, including the plenum, the sixteen swirling units and the whole chamber. A large volume is added around the chamber to mimic the atmosphere. To represent the entrainment of the atmospheric air induced by the mixture escaping from the chamber and by the room exhaust vent, a slow co-flow $\left(1 \mathrm{~m} . \mathrm{s}^{-1}\right)$ is added around the chamber. Standard Navier-Stokes characteristic boundary conditions [31] are used at the inlet, outlet and lateral walls of the system. The air injection is represented by eight injection patches at the end of tubes leading to the plenum with imposed mass flow rates, temperatures and gaseous species mass fractions. The pressure at the outlet is set to $101325 \mathrm{~Pa}$. All walls are considered adiabatic, with a slip condition on the liquid velocity and a wall law on the gaseous velocity. The sixteen fuel injection patches need a more detailed treatment since the internal geometry generating the swirled hollow cone is not known and therefore not simulated. In the absence of more information, the experimental profiles for the droplets velocities and the particle number density, measured $2 \mathrm{~mm}$ downstream the injector are directly imposed. Since the chosen description for the liquid phase is mono-disperse, imposing the experimental diameter distribution is not possible and the choice of the injected droplet diameter requires special care. A classical approach in the context of combustion [32] is to use the experimental Sauter Mean Diameter $(\mathrm{SMD}) D_{32}=\left(\sum_{\mathrm{N}} d^{3}\right) /\left(\sum_{\mathrm{N}} d^{2}\right)$ to represent the polydisperse spray. The value of the $D_{32}$ measured inside the spray $\left(D_{32}=20 \mu \mathrm{m}\right)$ is used as a first choice during the validation of the numerical set-up. A more detailed study is presented later on to derive a value that would better represent the spray, depending on the physical parameters that are identified as important to retrieve. The overall equivalence ratio considered for both simulation and experiment for this study is $\phi=0.89$ with a liquid mass flow rate for each injector $\dot{m}=0.111 \mathrm{~g} . \mathrm{s}^{-1}$.

Given the MICCA-Spray geometry, only one-eighth of the chamber, called in the following bi-sector, is considered with axially-periodic boundary conditions for the computation of the fully-developed flow of the initial un-reacted two-phase mixture. Once the flow of fresh mixture is converged, ignition can be initiated under conditions that correspond to the experimental situation. The mesh for the bisector comprises 36 million elements corresponding to 6.4 million nodes, while the full MICCA-Spray mesh comprises 288 million elements and 50 million nodes. 


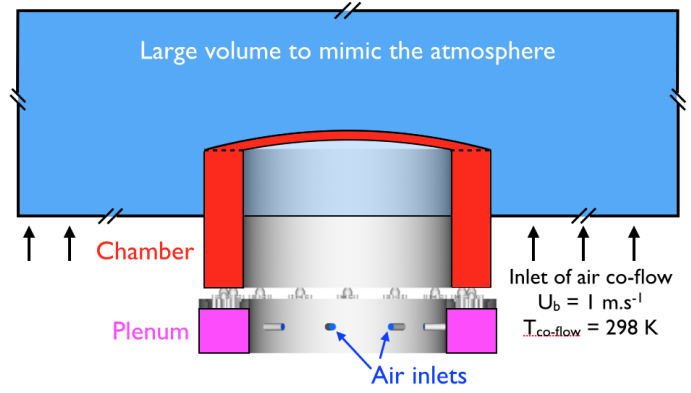

Fig. 3. Axial slice of the computational domain with the plenum colored in pink, the chamber in red and the outer atmosphere in blue. The axial co-flow is represented by black arrows.

\subsection{Numerical set-up validation on a single burner}

This section describes the various simulations and comparisons used to validate the numerical set-up and the modeling choices made at these stages before the simulation of the full MICCA-Spray geometry. The cylindrical shape of the annular chamber complicates most local measurements. It is thus convenient to consider a single swirling liquid fuel-air injector. The simpler geometry and smaller mesh of such a configuration enables the validation of the gaseous and twophase flow description.

\subsubsection{Single-burner geometry and mesh}

The single-burner geometry is shown in Fig. 4. The injector is identical to one of the sixteen units mounted on MICCA-Spray. No confinement is present to allow direct experimental measurements. The mesh for this simpler configuration comprises 17.5 million cells, corresponding to 3 million nodes, allowing to perform several LES at a reduced cost. A mesh convergence study has demonstrated the independence of the solution with respect to the mesh.

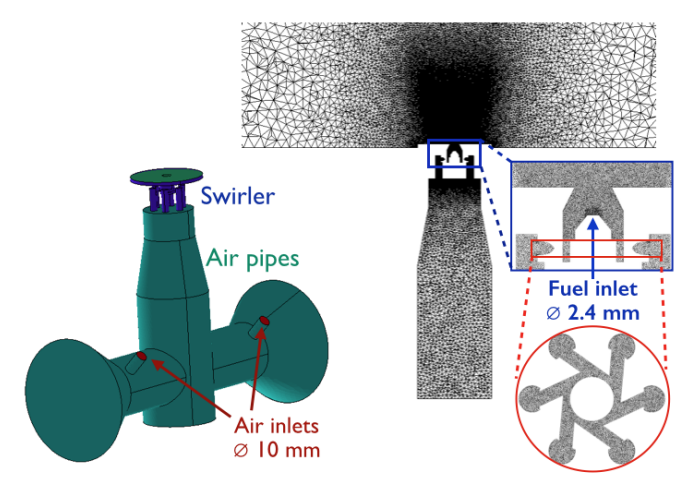

Fig. 4. Single burner computational domain with an axial slice of the mesh. The outer atmospheric domain is not shown.
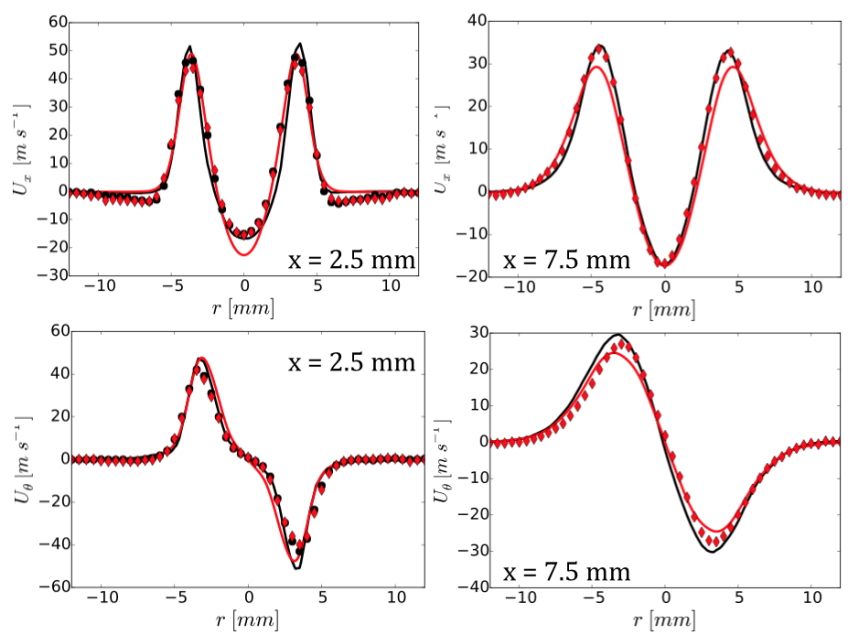

Fig. 5. Mean velocity profiles for the gas phase at $x=2.5 \mathrm{~mm}$ (left) and $x=7.5 \mathrm{~mm}$ (right) from the chamber backplane : axial velocity (top) and azimuthal velocity (bottom). Black curves represent the results from the gaseous simulation and the red curves the gaseous fields in the two-phase flow simulation. $-:$ Numerical results; $\bullet$ : Experimental gaseous velocity.

\subsubsection{Gaseous flow validation}

It is first important to verify that the numerical set-up suitably retrieves the gaseous phase behavior. This is accomplished by comparing gaseous velocity fields with experimental data in two cases: first, a study of the air stream when no liquid is being injected so as to validate the gaseous set of equations and models; second, liquid injection is included and the same comparisons are carried out in order to validate the gas-liquid interactions. Figure 5 shows the mean radial profiles of the axial and azimuthal gaseous velocities at two distances from the injection plane, for the case with gaseous and droplet injection. The symbols represent the experimental data while solid lines correspond to numerical results. The black color stands for the fully gaseous case (experimental data are only available at $x=2.5 \mathrm{~mm}$ ) and the red color for the two-phase one. For both cases, the numerical simulation is able to retrieve the main features of the swirled flow: the radial position and the magnitude of the central recirculation zone and the outer velocity peaks are accurately predicted. In addition, both experimental and numerical data show that the gaseous phase behaves similarly with or without the presence of droplets, indicating that the dispersed liquid phase has little impact on the gaseous phase velocity.

\subsubsection{Liquid phase validation}

To begin this validation, a first two-phase simulation is carried out with an injected droplet diameter $d_{l}^{\text {inj }}$ of liquid droplets set to the experimental value of the SMD: $d_{l}^{i n j}=$ $20 \mu \mathrm{m}$. Figure 6 shows the radial profile of the numerical mean tangential liquid velocity (in black line), compared to the mean velocities conditioned by droplets sizes (dashed lines), at $x=7.5 \mathrm{~mm}$. The experimental profiles are only 


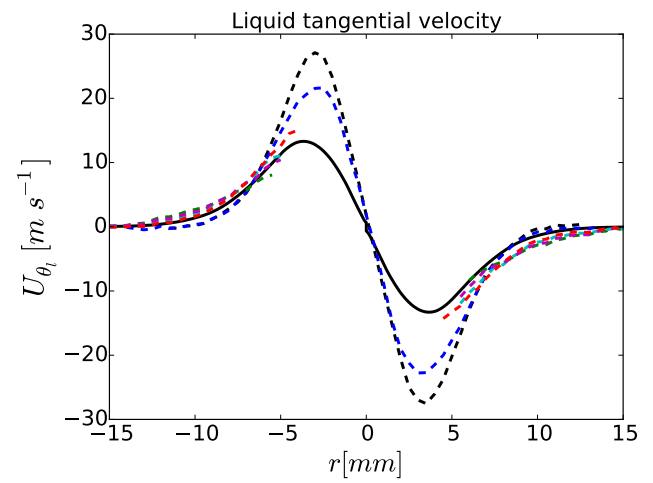

Fig. 6. Mean tangential velocity profile for the liquid phase at $x=$ $7.5 \mathrm{~mm}$. - : Numerical results; Experimental profiles: - -: $d_{l}=2-$ $3 \mu \mathrm{m} ;--: d_{l}=10-12 \mu \mathrm{m} ;--: d_{l}=20-23 \mu \mathrm{m} ;--: d_{l}=$ $23-36 \mu \mathrm{m} ;--: d_{l}=26-30 \mu \mathrm{m} .-\cdot: d_{l}=30-34 \mu \mathrm{m}$;

plotted when sufficient data is available to generate an average field. First of all, the comparison of the different experimental profiles shows a large variation in the behavior of the different droplet classes. The smaller droplets behave like the gaseous flow while the larger droplets have a more ballistic type of trajectory and are only present on the outskirts of the flow. Focusing on the two velocity peaks indicates that the simulation is able to retrieve the radial position of the spray. One also finds that the predicted velocity magnitude matches that of the corresponding simulated class (in red). From these observations one concludes that the simulation is able to correctly predict the dynamics of an injected class of droplets, but that the accuracy of representing the polydisperse spray with one that is mono-disperse has yet to be determined.

\subsection{Injection diameter selection}

Considering the mono-disperse description as a surrogate model of the polydisperse spray, this section presents a summary of a study carried out to define an optimal injected droplet diameter for the simulations with combustion [33]. In essence, one has to evaluate the impact of one or several parameters on the response of a complex system. This is accomplished by making use of uncertainty quantification (UQ) tools such as Polynomial Chaos Expansions (PCE) $[34,35]$ to derive a response surface of all LES results as a function of the injection diameter. The definition of a criterion adapted to the final purpose of light-round simulations then leads to an optimal value for the injected droplet diameter.

\subsubsection{Surface response generation}

In the present case, the injected droplet diameter $d_{l}^{i n j}$ is viewed as the only uncertain parameter that causes all physical and numerical fields to be uncertain. Through PCE, it is possible to approach these uncertain fields: any given field $\varphi$ can be written as $\varphi(x, \omega)$, where $x$ represents the deterministic parameters and $\omega$ the uncertain ones, and can be approxi- mated in terms of a polynomial decomposition:

$$
\begin{array}{r}
\varphi(x, \omega) \approx \sum_{k=0}^{N} a_{k}\left(x_{j}\right) P_{k}(\omega) \\
a_{k}(x)=\int_{\omega} \varphi(x, \omega) P_{k}(\omega) \pi(\omega) d \omega
\end{array}
$$

where $P_{k}$ is the Polynomial Chaos, $\pi(\omega)$ designates the distribution of the uncertain parameters $\omega, a_{k}(x)$ stand for the polynomial coefficients and $N$ defines the chosen polynomial order. An estimation of $\varphi(x, \omega)$ is therefore directly deduced from the values of the $a_{k}(x)$. A quadrature method can be used to estimate these values from a reduced number of evaluations. In the context of the present study, one evaluation corresponds to one LES simulation with a given injected droplet diameter $d_{l}^{i n j}$. The retained method, the ClenshawCurtis nested quadrature rule enables to limit the number of evaluations for several quadrature levels [36]. Comparisons between results from quadrature levels 3 and 4, respectively corresponding to 9 and 17 LES, show that the generated response surfaces are close, indicating a good convergence for the results and that a quadrature level of 3 can be considered accurate.

\subsubsection{Diameter selection}

The strength of the present method is that once all simulations identified by the quadrature rule have been converged and average fields have been recovered, the reconstruction by the PCE of response surfaces yields the variation of any physical or numerical field, at any point in space, according to the injected droplet diameter. A criterion can then be defined to extract an optimal value. Experimental visualizations of the light-round sequence in MICCA-Spray show a flame front propagation in the unburnt two-phase flow. In the case of gaseous propagating flames, a key quantity is the laminar burning velocity $S_{L}$. In order to account for the presence of droplets, Ballal and Lefebvre [37] proposed an expression for a two-phase laminar burning speed $S_{l}^{t-p}$, that has been validated numerically in [38]:

$$
S_{l}^{t-p}=\alpha_{g}\left[\frac{C_{3}^{3} \rho_{l} D_{32}^{2}}{8 C_{1} \rho_{g} \ln (1+\mathrm{B})}+\frac{\alpha_{g}^{2}}{S_{L}^{2}}\right]^{-0.5}
$$

with $\alpha_{g}$ the gas thermal diffusivity, $\rho_{l}$ and $\rho_{g}$ the liquid and gaseous densities, B the Spalding transfer number and $S_{L}$ the gaseous laminar burning velocity. This expression accounts for polydispersion through the coefficients $C_{1}=D_{20} / D_{32}$ and $C_{3}=D_{30} / D_{32}$, which are both equal to unity in the case of a mono-disperse spray. The expression of $S_{l}^{t-p}$ can be evaluated from the experimental polydisperse data, yielding $S_{l \text { exp }}^{\text {poly }}$, and compared to the same expression determined from mono-disperse simulation data, $S_{l P C E}^{t-p}\left(d_{l}^{i n j}\right)$, as a continuous function of $d_{l}^{i n j}$ thanks to the reconstructed response surfaces. This is done in Fig. 7 which shows the relative error 


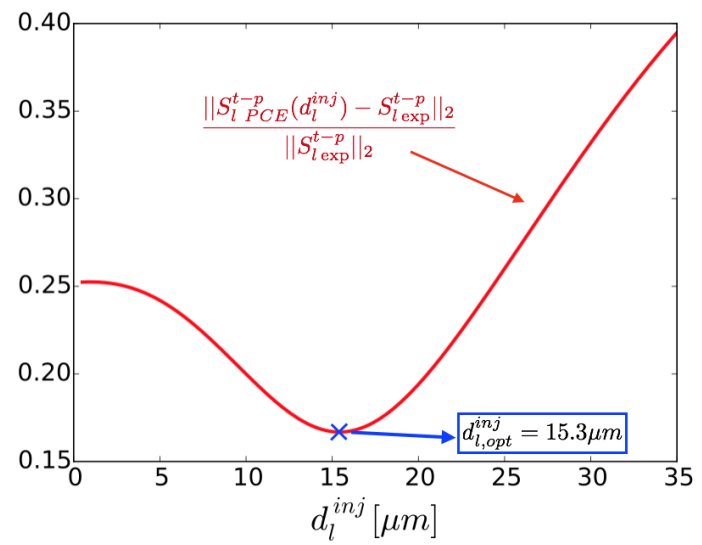

Fig. 7. Relative error in the numerical approximation of the twophase laminar burning velocity $S_{l}^{t-p}$.

$\left\|S_{l \text { PCE }}^{t-p}\left(d_{l}^{\text {inj }}\right)-S_{\text {l exp }}^{\text {poly }}\right\|_{2} /\left\|S_{l \text { exp }}^{\text {poly }}\right\|_{2}$. This defines an optimal value for the injected droplet diameter of about $15.3 \mu \mathrm{m}$ that will reproduce the burning velocity. For this diameter, the relative error on the burning velocity is about $16 \%$, which remains noticeable and highlights the limitations that arise when a polydisperse spray is represented by a mono-disperse approach.

\section{Light-round results and discussion}

The ignition is experimentally initiated by means of a plug generating a series of sparks until a flame kernel forms with sufficient energy so that it is able to propagate. This first phase involving complex non-equilibrium physics is not examined here. The present study focuses on the case where the plug has succeeded in creating a stable kernel, that is numerically represented by a sphere of burnt gases placed in the vicinity of the first injector in a converged cold flow solution. To assert the simulation quality, the temporal evolution of the flame is first compared with direct visualizations recorded by the CMOS camera during the light-round. Snapshots at different instants are examined to compare experimental and numerical flame geometries.

\subsection{Cold flow simulation for MICCA-Spray}

As indicated previously, the ignition is initiated once the cold two-phase flow is fully-developed in the chamber. While such a condition is easily met experimentally by waiting a few seconds, it is not possible to simulate this time duration, even in the bi-sector configuration. A criterion has to be defined to check that uniform mixture conditions are approximately met. Figure 8 shows the temporal evolution of the mean gas equivalence ratio in the chamber (shown as black dots), which appears to evolve slowly compared to the convective time in the chamber, that is of the order of ten milliseconds. This indicates that even though the velocity fields are converged quite rapidly, some recirculation regions appear between the swirlers that trap most of the droplets, creating a transient accumulation of fuel mass fraction that only

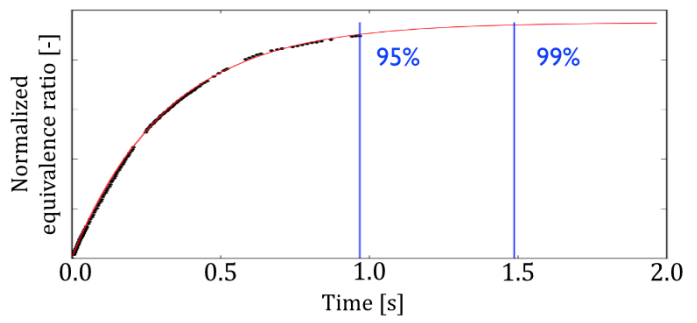

Fig. 8. Global equivalence ratio inside the bi-sector domain. $\bullet$ : Numerical values; -: Exponential fit

slowly reaches the exit of the chamber. The red curve represents a fitting on $a$ and $\lambda$ in the function $a\left(1-e^{-\lambda x}\right)$. For the present study, the cold flow field was considered converged when the global equivalence ratio reached $95 \%$ of the estimated final value. To obtain $99 \%$ would have necessitated $0.5 \mathrm{~s}$ more of simulated physical time, which was unaffordable considering the computational resources available.

\subsection{Flame geometry and heat release}

The experimental images of the propagating flame front generated by the CMOS camera, initially in levels of grey, are represented on a scale of colors from yellow to red for better clarity. It is worth noting that the globally lean operating conditions lead to blue flames without noticeable soot emission. To be able to draw comparisons, an isosurface of the progress variable $c=0.9$ defined as:

$$
c=\frac{Y_{\mathrm{CO}_{2}}+Y_{C O}}{Y_{C O_{2}}^{e q}+Y_{C O}^{e q}}
$$

is used in the numerical simulations to represent the flame front. This isosurface is colored by the axial gaseous velocity, from $-10 \mathrm{~m} . \mathrm{s}^{-1}$ in yellow to $25 \mathrm{~m} . \mathrm{s}^{-1}$ in black. For better visualization, isosurfaces of the axial gaseous velocity at $u=25 \mathrm{~m} \cdot \mathrm{s}^{-1}$ are added to highlight the presence of each injector as well as the interaction between the flame and the flow field. To avoid mesh refinement in the atmospheric domain surrounding the MICCA-Spray chamber, combustion is not represented numerically beyond the chamber exit plane, which means that the flame plume that is observed exiting the chamber in the experiments is not present in the simulations. Figure 9 displays instantaneous views of experimental and numerical sequences during the process. Experimental images and numerical visualizations are synchronized using the first burner ignition as reference, which corresponds to a simulated physical time of around $10 \mathrm{~ms}$. On the global level, it appears clearly that the simulation is able to retrieve the main aspects of the process. After initiation (Fig. 9, top left), the kernel grows until the first swirler is ignited. The flame then propagates on both sides of the chamber forming an arch that rapidly expands (Fig. 9, middle left) until its top reaches the exit of the chamber, where the flame splits in two fronts (Fig. 9, bottom left). These fronts then travel in their respective chamber domain (Fig. 9, right). As observed ex- 

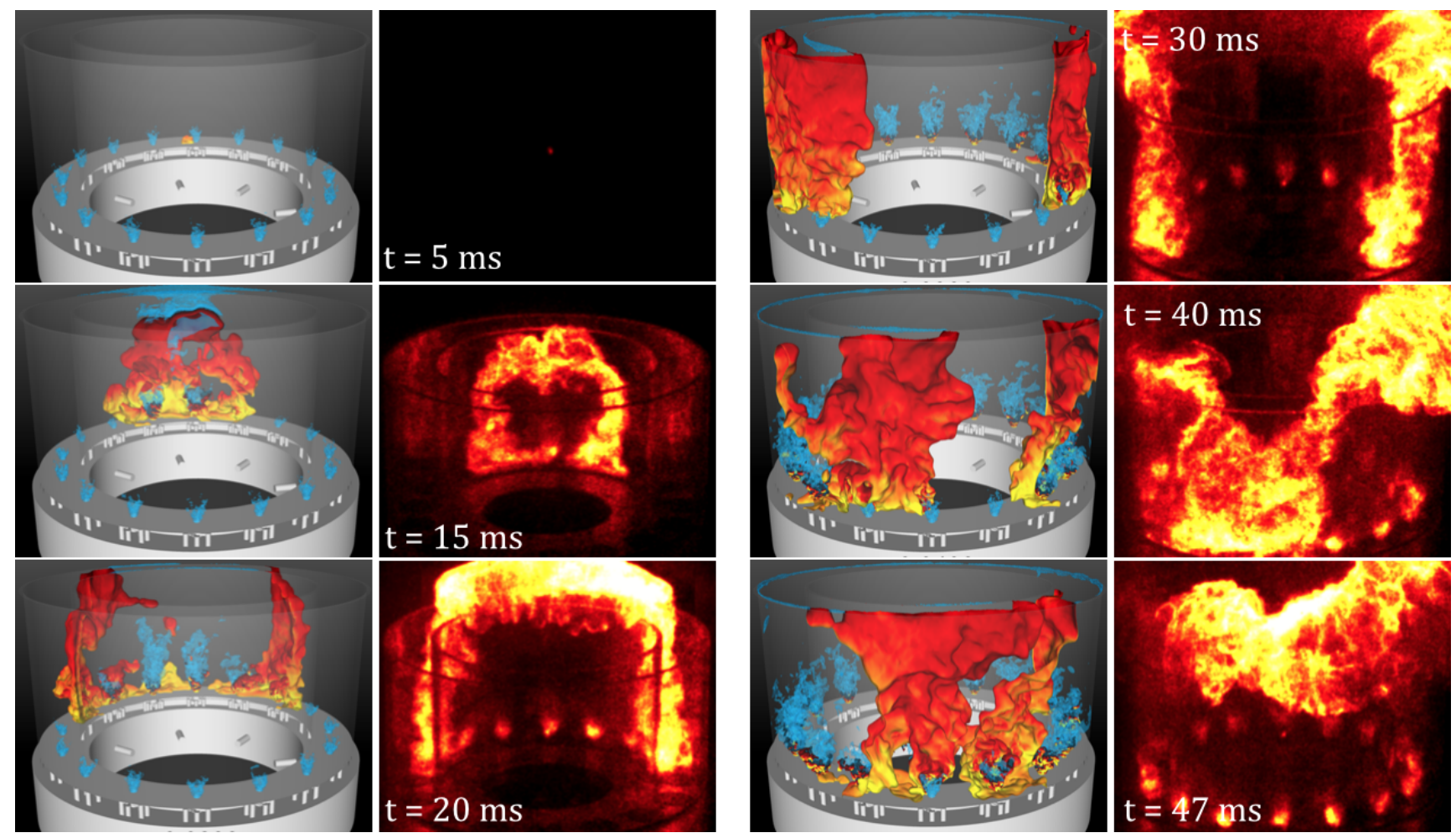

Fig. 9. Comparison between experimental and numerical flame configurations at six initial instants during the light-round process, $t=5 \mathrm{~ms}$ (top left), $t=15 \mathrm{~ms}$ (middle left), $t=20 \mathrm{~ms}$ (bottom left), $t=30 \mathrm{~ms}$ (top right), $t=40 \mathrm{~ms}$ (middle right) and $t=47 \mathrm{~ms}$ (bottom right).

perimentally, the two phase light-round is qualitatively similar to what was found in the premixed gaseous case [5,6], with a more pronounced deceleration in the final stages of the simulation leading to a slight delay in the final merging.

Another way to examine the flame evolution is to consider the temporal records of the integrated heat release rate over the whole chamber. This is done experimentally by integrating the flame light intensity on each image. While the relationship between heat release rate and light intensity is well established for premixed flames, this is not true in general for two-phase flames which can exhibit complex reaction layers with premixed and non-premixed combustion modes. The analysis of the simulated flame structure (detailed later) reveals that the reaction zone mainly behaves like a premixed flame front. This observation justifies the use of light intensity to characterize the rate of heat release in the spray flame. The two-phase nature of the flow nonetheless prevents a quantitative comparison. Besides, the visual integration of the light emission can introduce some error when some parts of the flame hide one another. The presence of the quartz tube also leads to a damping of the light intensity, especially during the first instants when the flame is in the back of the chamber. The numerical integrated heat release and the integrated experimental light intensity are then both normalized by their respective maximum and compared qualitatively. These two quantities are plotted in Figure 10. The comparison of the different snapshots shows that some common features can be retrieved between experiment and simulation. As in previous studies [5], several stages can be defined in the light-round process from the evolution of the integrated heat release, as indicated in Fig. 10. It is worth noting that the stages identified in this figure are defined with reference to the experimental data:

(I) During the first instants after the initiation of ignition, the kernel is convected by the flow exhausted by the first swirler and distorted by turbulence. Once it meets an area with favorable conditions, the flame starts to propagate and the volume of burnt gases is suddenly augmented, leading to the sharp increase in the integrated heat release rate.

(II) The flame front takes the form of an arch rapidly expanding sideways and towards the neighboring burners and the exit plane of the chamber. During this phase, the integrated heat release rate increases in a quasi-linear fashion, both numerically and experimentally. The end of this phase is characterized by a sudden break in the slope in both curves and a local maximum, present in numerical and experimental curves and exactly synchronized.

(III) Two flame fronts are created that propagate in the lightround process from burner to burner. Also during this phase, the integrated heat release rate increases at a constant rate that is at first well predicted numerically. However, once each front has crossed a quarter of the chamber, the numerically integrated heat release rate strongly differs from the experimental data, in terms of slope as well as tendency, since a maximum is reached in the simulation at this point and not in the experiment. The snapshots in Fig. 9 indicate that at that time, the simulated flame begins to propagate at a slightly slower pace than in the experiments. 


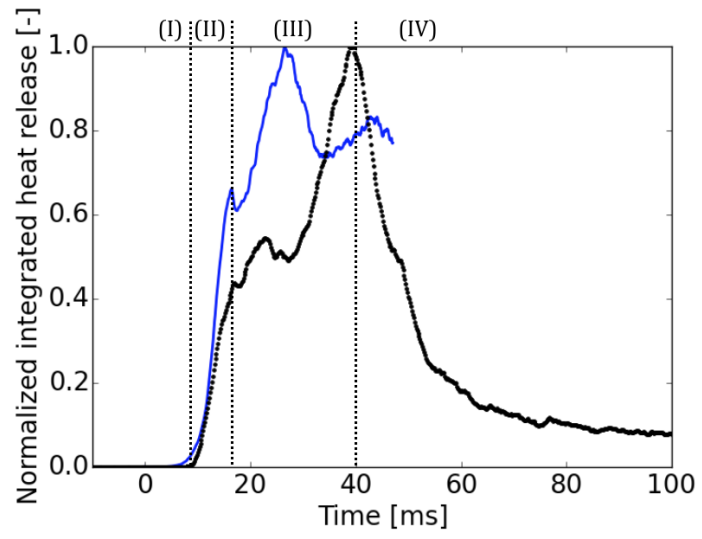

Fig. 10. Time evolution of the integrated heat release rate over the whole chamber (blue-line) and the experimental integrated heat release over the whole chamber symbols, normalized by their respective maximum.

(IV) During the final phase where head on collision takes place the proximity of the two reactive fronts creates an interaction that influences their shape. The effects of the upstream blast generated by each flame front is further investigated in the following.

As already mentioned, only combustion taking place inside the chamber is considered in the simulation. Experimentally, it is not possible to separate the combustion in the chamber from that taking place in the plume of gases burning outside the chamber. This constitutes a source of error since the plume light emission is especially important during the merging of the fronts, and is therefore taken into account in the normalization of the integrated light intensity and impacts all temporal values. The discrepancy that appears during the transition between phases (II) and (III) could be explained by the flame being partly hidden behind the two quartz walls at the same time, enhancing the decrease of light emission. However, a maximum is reached numerically before the merging, contrary to the experiments, which remains to be explained.

\subsection{Propagation velocity and light-round duration}

During the propagation process, a leading point can be identified for each flame front. Two leading points are therefore defined, one for each half of the chamber (see Fig 2), and their angular position with respect to the original kernel, and absolute velocity, can now be examined. Figure 11 displays the evolution of the absolute velocities of these leading points, for $H+$ (in red) and $H$ - (in blue) as a function of their angular position. The sudden jumps that appear are linked to the fact that several fronts may compete locally and overtake one another at times, generating discontinuities. The observed order of magnitude of the flame fronts velocity $(\approx 20 \mathrm{~m} / \mathrm{s})$ is much larger than the one explained from turbulent wrinkling effects. Such a value is in fact due to the effect of burnt gases volumetric expansion (detailed later) and the corresponding induced flow in the fresh gases. During the propagation phase (III), the leading point velocity decreases in a quasi linear fashion, highlighting the trend previously observed in the snapshots. These two leading points angular positions are used to determine the light-round duration $\tau_{l}$, defined as the delay between the ignition of the injector in S1 (see Fig. 2) and the head on collision of the flame fronts, which yields a light-round duration of $\tau_{l}=29.3 \mathrm{~ms}$.

Figure 12 presents light-round durations for a series of experimental runs. The durations are estimated from the CMOS camera images by identifying the two instants when the flame reaches the injector in S1 and when the flame fronts merge on the other side of the chamber, knowing the physical time between two consecutive images. Two cases for the ignition are displayed here, depending on the temperature of the walls. It appears that, similarly to the gaseous cases [5], the ignition delay varies by a large amount between a "cold case", where the walls are at ambient temperature, and a "hot case", where the chamber has been operated for some time. For all the experimental points, the target value for the equivalence ratio was the same as for the simulation $(\phi=0.89)$, but some variability is introduced by the flowrate meters and the equivalence ratios reported correspond to actual flowrates measured when ignition is initiated. As was observed in [11], when the walls are pre-heated and on the considered range, the light-round duration decreases linearly when the equivalence ratio increases. A first observation is that most of the experimental shots correspond to an equivalence ratio higher than the one in the simulation, which is the targeted nominal case. The difference in light-round durations between simulation and experiment can therefore be partly explained by the difference of equivalence ratio and the observed trend. A linear interpolation of the different available experimental shots (blue dashed curve in Fig. 12) gives an estimate of the light-round duration for the simulated operating point. The value for the simulation is also reported in Fig. 12, indicating that the light-round duration is close to the experimental prediction, with a slight overestimation ranging between 2.5 and $3 \mathrm{~ms}$, which corresponds to an error of $10 \%$.

\subsection{Preliminary analysis}

It is now informative to consider the core of the lightround phase, when two separate fronts propagate in their respective sides of the annular chamber. This can be observed by considering a cylindrical cut in the chamber and unfolding this surface to ease the analysis (see Fig. 13 and 14). The unfolded cylinder edges correspond to the location of the first ignited injector, so that the two flame fronts are seen propagating towards the center of the unfolded cylinder surface. The flame fronts are represented by two iso-lines of the progress variable, at $c=0.9$ and $c=0.1$. Figure 13 presents the field of droplet diameter at several moments during the process. These figures show that in the fresh mixture part of the chamber, once injected in the chamber, the droplets (initially having a diameter of $15.0 \mu \mathrm{m}$ ) begin to evaporate, and exit the chamber with a diameter of $8 \mu \mathrm{m}$, indicating that the 


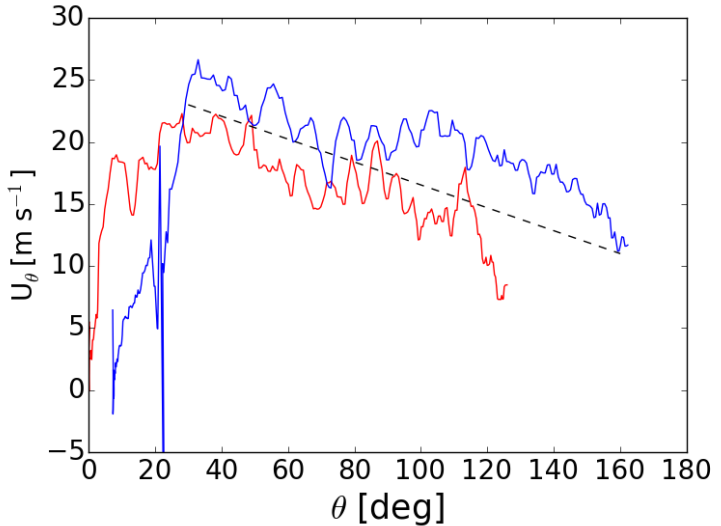

Fig. 11. Evolution of the leading point velocity as a function of the angular position for the flame propagating in the $\mathrm{H}+$ side (red curve) and in the $\mathrm{H}$ - side (blue curve). A linear approximation of the azimuthal velocity is also shown as a dashed line.

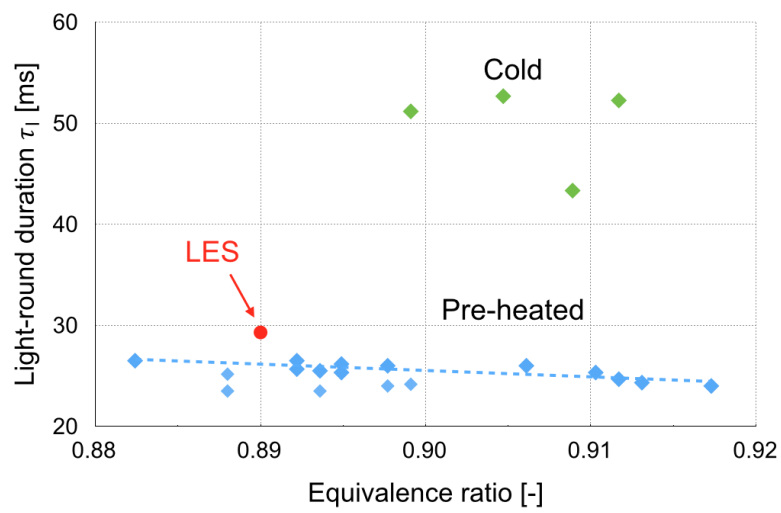

Fig. 12. Light-round durations as a function of the global equivalence ratio. Diamond symbols stand for experimental results. The duration predicted by the simulation is represented by a red circle.

flame does propagate in a two-phase flow and that the liquid phase is not totally vaporized. Figure 14 shows the fields of gaseous equivalence ratio for the same instants as Fig. 13. During the light-round phase, this field appears to be globally homogeneous outside of the swirler zones. Examining more closely the vicinity of the flame fronts as shown in the zooms in Fig. 15, it appears that at the first iso-line, corresponding to $c=0.1$, the droplet diameter is quickly reduced to zero, indicating that the liquid phase is vaporized by the flame so that no droplets are left inside the flame reaction zone. In the same area, an increase of gaseous equivalence ratio is logically observed, creating locally richer zones (in red) in front of the flame. In order to analyse the local combustion regime and using the methodology proposed in [39] and [40], the instantaneous Takeno's flame index (TI) is calculated and displayed in Fig. 16 at $t=30 \mathrm{~ms}$. To highlight the combustion areas, TI was multiplied by the absolute value of the heat

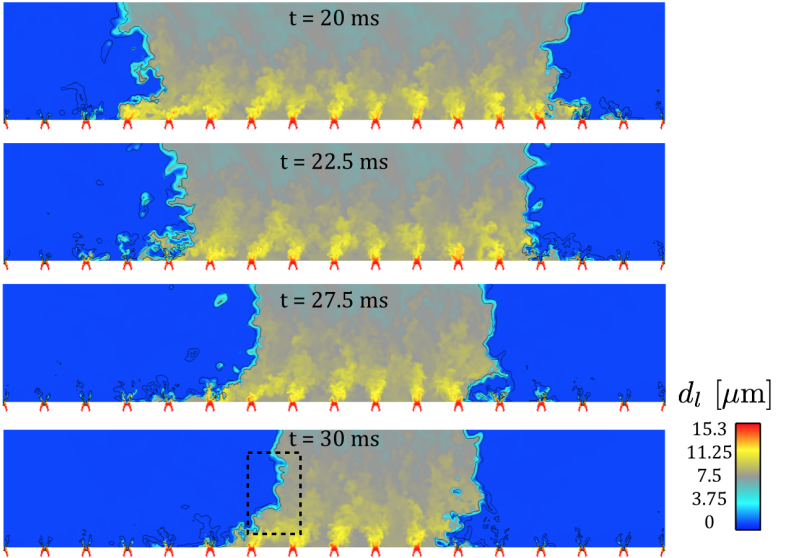

Fig. 13. Visualization of the local droplet diameter on a cylinder of radius $r=0.175 \mathrm{~m}$ unfolded on a plane surface. The lateral sides of the unfolded cylinder correspond to the location of the first ignited injector. A zoom of the dashed rectangle is shown in Fig.15.

release rate:

$$
\mathrm{TI} \cdot|\mathrm{HR}|=\frac{\nabla Y_{C_{7} H_{16}} \cdot \nabla Y_{O_{2}}}{\left|\nabla Y_{C_{7} H_{16}} \cdot \nabla Y_{O_{2}}\right|} \cdot|\mathrm{HR}|
$$

Positive values indicate premixed combustion areas while negative values correspond to non-premixed reaction layers. Figure 16 shows that the combustion is dominated by a premixed regime.

Figure 17 gives the field of tangential velocity, calculated so that for each side of the chamber, it is positive (and shown in red) when oriented towards the merging point. An increase of the tangential velocity appears clearly near both flame fronts, which indicates that the fresh gas velocity is modified before the flame reaches this region. This is due to the burnt gases volumetric expansion. This specific flow in fresh gases induced by both propagating flame fronts is a specific phenomenon of the light-round and shows the added benefit of studying the annular enclosure compared to a simpler geometry.

Examining more closely the evolution of the value of the droplet diameter at the exit of the chamber, one observes a slight decrease between the first and last instants displayed in Fig. 13. This is consistent with the global increase of equivalence ratio that can also be seen in Fig. 14. Such a variation in 10 milliseconds is not explained from the previously observed characteristic time of the fuel vapor accumulation (of the order of a second). A possible explanation is that the flow generated in the cold gases by the propagation of the flame fronts enhances evaporation. A two-way coupling mechanism between the flame propagation and the two-phase mixture located ahead probably plays an essential role in two-phase light-round that needs to be further investigated. 


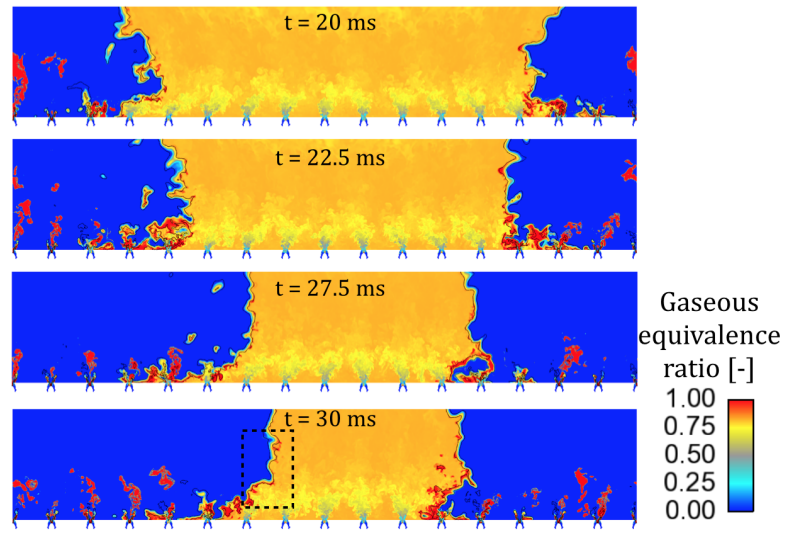

Fig. 14. Visualization of the local gaseous equivalence ratio on an unfolded cylinder of radius $r=0.175 \mathrm{~m}$. The lateral sides of the unfolded cylinder correspond to the location of the first ignited injector. A zoom of the dashed rectangle is shown on Fig.15.

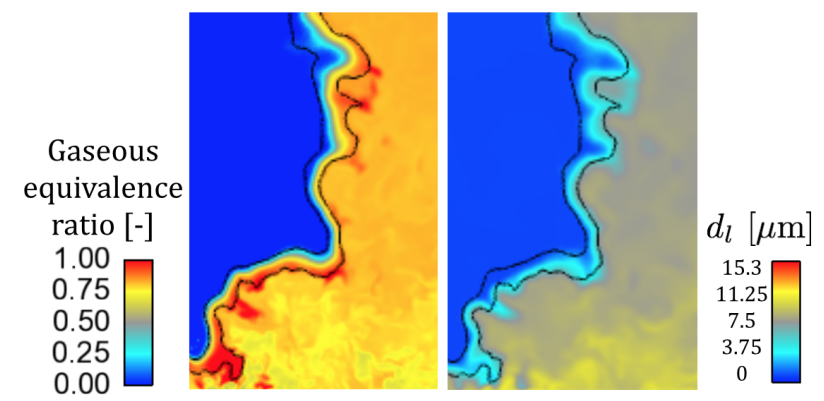

Fig. 15. Zoom on the left flame front (see Fig.13 and 14 for the zoom area) of the equivalence ratio field (left) and the droplet diameter (right) at $t=30 \mathrm{~ms}$.

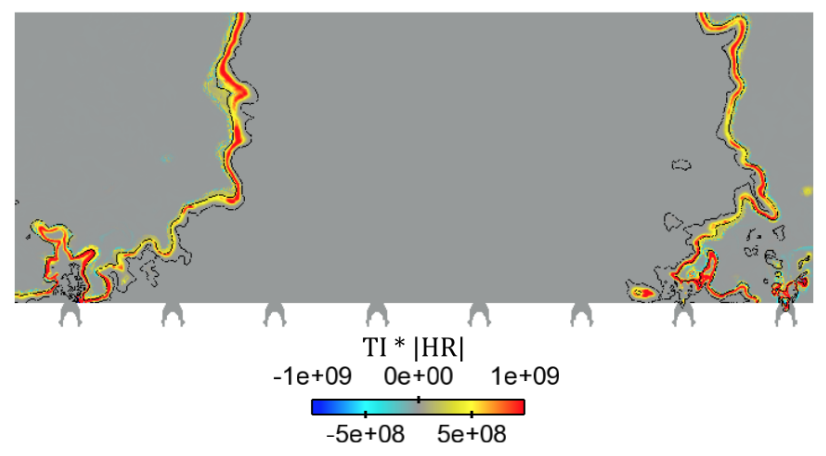

Fig. 16. Field of Takeno index, multiplied by the absolute value of heat release at $t=30 \mathrm{~ms}$. Positive values indicate premixed combustion regime areas while negative values indicate diffusion regime areas.

\section{Conclusion}

The present investigation is concerned with the large eddy simulation of the light-round process in an annular combustor equipped with multiple swirl spray injectors. Results of calculations are compared with experiments. This is

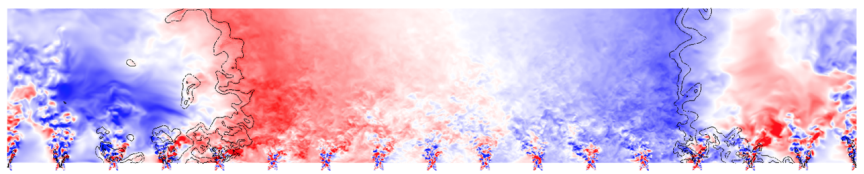

Fig. 17. Visualization of the tangential velocity at one instant in time $t=27.5 \mathrm{~ms}$. The blue color corresponds to a velocity $-20 \mathrm{~m} . \mathrm{s}^{-1}$ while the red pertains to a value of $20 \mathrm{~m} . \mathrm{s}^{-1}$. Velocities are counted positive from left to right.

accomplished by making use of a model scale device allowing full optical access to the combustion region. This experimental system is dimensionally similar to a typical helicopter gas turbine combustor and is operated in a liquid injection mode in an effort to achieve a better representativity of aeroengine combustors. The detailed visualizations of the flame during light-round allow precise comparisons between experiments and simulations. The LES simulation accounts for the liquid disperse phase with an Eulerian-Eulerian monodisperse approach. After validation of the different modeling choices, detailed comparisons are presented. The global dynamics and the flame geometry are found to be similar and simulation are in agreement with experiments but with a slight overestimation of the light-round delay. The macroscopic evolution of the flame is generally well retrieved numerically, again with some differences in the evolution of the integrated heat release rate. Finally, an analysis of the fresh gas behavior indicates the presence of a specific flow, induced by the two propagating fronts, that modifies the gaseous and liquid fields ahead of the flames. The differences observed between simulation and experiment are not fully explained, though the selected models, such as the monodisperse approach or the two-step chemical scheme, represent simplifications that influence the quality of predictions. In the future, further analysis is needed to extract a better understanding of the sequence, and of the impact of the spray injection. Other light-round simulations will also be performed with different combustion models and spray modeling approaches in order to quantify their impact.

\section{Acknowledgements}

This work was granted access to the HPC resources of TGCC and IDRIS under the allocation 2016-020164 made by GENCI. This work was also supported by CNRS, SAFRAN Tech and by the French National Research Agency (Timber project ANR14-CE23-0009-01).

\section{References}

[1] Lefebvre, A., and Ballal, D. R., 2010. Gas Turbine Combustion. Taylor and Francis.

[2] Boileau, M., Staffelbach, G., Cuenot, B., Poinsot, T., and Bérat, C., 2008. "Large eddy simulation of an ignition sequence in a gas turbine engine". Combustion and Flame, 154(1-2), pp. $2-22$.

[3] Barré, D., Esclapez, L., Cordier, M., Riber, E., Cuenot, B., Staffelbach, G., Renou, B., Vandel, A., Gicquel, L. Y., and Cabot, G., 2014. "Flame propagation in aeronautical swirled multi-burners: Experi- 
mental and numerical investigation". Combustion and Flame, 161(9), pp. $2387-2405$.

[4] Bourgouin, J.-F., Durox, D., Schuller, T., Beaunier, J., and Candel, S., 2013. "Ignition dynamics of an annular combustor equipped with multiple swirling injectors". Combustion and Flame, 160(8), pp. 1398 $-1413$.

[5] Philip, M., Boileau, M., Vicquelin, R., Schmitt, T., Durox, D., Bourgouin, J.-F., and Candel, S., 2015. "Simulation of the Ignition Process in an Annular Multiple-Injector Combustor and Comparison With Experiments". Journal of Engineering for Gas Turbines and Power (ASME), 137(3).

[6] Philip, M., Boileau, M., Vicquelin, R., Riber, E., Schmitt, T., Cuenot, B., Durox, D., and Candel, S., 2015. "Large eddy simulations of the ignition sequence of an annular multiple-injector combustor". Proceedings of the Combustion Institute, 35(3), pp. 3159-3166.

[7] Worth, N. A., and Dawson, J. R., 2013. "Self-excited circumferential instabilities in a model annular gas turbine combustor: Global flame dynamics". Proceedings of the Combustion Institute, 34(2), pp. 31273134.

[8] Worth, N. A., and Dawson, J. R., 2013. "Modal dynamics of selfexcited azimuthal instabilities in an annular combustion chamber". Combustion and Flame, 160(11), 11, pp. 2476-2489.

[9] Bach, E., Kariuki, J., Dawson, J. R., Mastorakos, E., and joerg Bauer, H., 2013. "Spark ignition of single bluff-body premixed flames and annular combustors". In 51th AIAA Aerospace Science Meeting, Grapevine (Dallas/Ft. Worth Region), Texas.

[10] Machover, E., and Mastorakos, E., 2016. "Spark ignition of annular non-premixed combustors". Experimental Thermal and Fluid Science, 73, 5, pp. 64-70.

[11] Prieur, K., Durox, D., Beaunier, J., Schuller, T., and Candel, S., 2017. "Ignition dynamics in an annular combustor for liquid spray and premixed gaseous injection.”. Proceedings of the Combustion Institute, 36(3), pp. 3717-3724.

[12] Fox, R. O., 2012. "Large-Eddy-Simulation Tools for Multiphase Flows". Annual Review of Fluid Mechanics, 44, pp. 47-76.

[13] Sanjosé, M., Senoner, J. M., Jaegle, F., Cuenot, B., Moreau, S., and Poinsot, T., 2011. "Fuel injection model for Euler-Euler and EulerLagrange large-eddy simulations of an evaporating spray inside an aeronautical combustor". Int. Journal of Multiphase Flow, 37(5), 6, pp. 514-529.

[14] Senoner, J. M., Sanjosé, M., Lederlin, T., Jaegle, F., García, M., Riber, E., Cuenot, B., Gicquel, L., Pitsch, H., and Poinsot, T., 2009. "Eulerian and Lagrangian Large-Eddy Simulations of an evaporating twophase flow". Comptes Rendus Mécanique, 337(6-7), pp. 458-468.

[15] Chrigui, M., Gounder, J., Sadiki, A., Masri, A. R., and Janicka, J., 2012. "Partially premixed reacting acetone spray using les and fgm tabulated chemistry". Combustion and Flame, 159(8), 8, pp. 27182741.

[16] Jones, W. P., Lyra, S., and Navarro-Martinez, S., 2012. "Numerical investigation of swirling kerosene spray flames using large eddy simulation". Combustion and Flame, 159(4), pp. 1539-1561.

[17] Luo, K., Pitsch, H., Pai, M. G., and Desjardins, O., 2011. "Direct numerical simulations and analysis of three-dimensional n-heptane spray flames in a model swirl combustor". Proceedings of the Combustion Institute, 33(2), pp. 2143-2152.

[18] Garcia, M., 2009. "Development and validation of the Euler-Lagrange formulation on a parallel and unstructured solver for large-eddy simulation". PhD thesis, Institut National Polytechnique de Toulouse.

[19] Schönfeld, T., and Rudgyard, M., 1999. "Steady and unsteady flow simulations using the hybrid flow solver avbp". AIAA Journal, 37(11), pp. 1378-1385.

[20] Colin, O., and Rudgyard, M., 2000. "Development of high-order taylor-galerkin schemes for LES". Journal of Computational Physics, 162(2), pp. $338-371$.

[21] Nicoud, F., and Ducros, F., 1999. "Subgrid-scale stress modelling based on the square of the velocity gradient tensor". Flow, Turbulence and Combustion, 62(3), pp. 183-200.

[22] Boileau, M., Pascaud, S., Riber, E., Cuenot, B., Gicquel, L. Y. M., Poinsot, T. J., and Cazalens, M., 2008. "Investigation of two-fluid methods for large eddy simulation of spray combustion in gas turbines". Flow Turbulence and Combustion, 80(3), pp. 291-321.

[23] Abramzon, B., and Sirignano, W., 1989. "Droplet vaporization model for spray combustion calculations". International Journal of Heat and Mass Transfer, 32(9), pp. $1605-1618$.

[24] Shum-Kivan, F., Santiago, J. M., Verdier, A., Riber, E., Renou, B., Cabot, G., and Cuenot, B., 2016. "Experimental and numerical analysis of a turbulent spray flame structure". Proceedings of the Combustion Institute, 36(2), pp. 2567-2575.

[25] Paulhiac, D., 2015. "Modélisation de la combustion d'un spray dans un bruleur aéronautique". PhD thesis, INP Toulouse.

[26] Franzelli, B., Riber, E., Sanjosé, M., and Poinsot, T., 2010. "A twostep chemical scheme for kerosene-air premixed flames". Combustion and Flame, 157(7), pp. $1364-1373$

[27] Butler, T., and O'Rourke, P., 1977. "A numerical method for two dimensional unsteady reacting flows". 16th International Symposium on Combustion, 16(1), pp. 1503 - 1515.

[28] Colin, O., Ducros, F., Veynante, D., and Poinsot, T., 2000. "A thickened flame model for large eddy simulations of turbulent premixed combustion". Physics of Fluids, 12(7), pp. 1843-1863.

[29] Legier, J. P., Poinsot, T., and Veynante, D., 2000. "Dynamically thickened flame les model for premixed and non-premixed combustion". In Proc. Summer Program, Center for Turbulence Research, NASA Ames/Stanford Univ.

[30] Charlette, F., Meneveau, C., and Veynante, D., 2002. "A power-law flame wrinkling model for LES of premixed turbulent combustion part I: non-dynamic formulation and initial tests". Combustion and Flame, 131(1-2), pp. $159-180$.

[31] Poinsot, T., and Lele, S., 1992. "Boundary conditions for direct simulations of compressible viscous flows". Journal of Computational Physics, 101(1), pp. $104-129$.

[32] Lefebvre, A., 1989. Atomization and Sprays. Taylor and Francis.

[33] Lancien, T., Dumont, N., Prieur, K., Durox, D., Candel, S., Gicquel, O., and Vicquelin, R., 2016. "Uncertainty quantification of injected droplet size in mono-dispersed eulerian simulations". In International Conference on Multiphase Flow, May 22 to 27, Firenze, Italy.

[34] Reagana, M. T., Najm, H. N., Ghanem, R. G., and Knio, O. M., 2003. "Uncertainty quantification in reacting-flow simulations through nonintrusive spectral projection". Combustion and Flame, 132(3), pp. 545-555.

[35] Xiu, D., and Karniadakis, G. E., 2002. "The wiener-askey polynomial chaos for stochastic differential equations". SIAM journal on scientific computing, 24(2), pp. 619-644.

[36] Khalil, M., Lacaze, G., Oefelein, J. C., and Najm, H. N., 2015. "Uncertainty quantification in les of a turbulent bluff-body stabilized flame". Proceedings of the Combustion Institute, 35(2), pp. 11471156.

[37] Ballal, D., and Lefebvre, A., 1981. "Flame propagation in heterogeneous mixtures of fuel droplets, fuel vapor and air". Symposium (International) on Combustion, 18(1), pp. 321 - 328. Eighteenth Symposium (International) on Combustion.

[38] Neophytou, A., and Mastorakos, E., 2009. "Simulations of laminar flame propagation in droplet mists". Combustion and Flame, 156(8), pp. $1627-1640$.

[39] Yamashita, H., Shimada, M., and Takeno, T., 1996. "A numerical study on flame stability at the transition point of jet diffusion flames". Symposium (International) on Combustion, 26(1), pp. $27-34$.

[40] Reveillon, J., and Vervisch, L., 2005. "Analysis of weakly turbulent dilute-spray flames and spray combustion regimes". Journal of Fluid Mechanics, 537, pp. 317-347. 